01

\title{
Электродинамика фотона и его структура как сгустка одного из многих возможных состояний электромагнитного поля
}

\author{
(С) А.И. Лаптухов \\ Институт земного магнетизма, ионосферы и распространения радиоволн им. Н.В. Пушкова РАН, \\ 108840 Троицк, Москвовская обл., Россия \\ e-mail: a.laptukhov@mail.ru
}

(Поступило в Редакцию 13 февраля 2017 г.)

Исходя из фундаментальных законов сохранения показано, что электромагнитное поле в общем случае даже в вакууме $(\rho=0, \mathbf{j}=0)$ описывается (как и в среде) четырьмя векторами $\mathbf{D}, \mathbf{E}, \mathbf{B}, \mathbf{H}$ с разными уравнениями состояния („материальными уравнениями“): линейными для электромагнитных волн и нелинейными для фотонов и частиц. Найдено уравнение, которое описывает разные состояния электромагнитного поля, т.е. различные, но не произвольные, связи между векторами поля $\mathbf{E}, \mathbf{H ,} \mathbf{D}$ и В. Показано, что электромагнитная волна и фотон - это два разных состояния электромагнитного поля, имеющие две разные функции зависимости плотности энергии от векторов поля. Получены частные аналитические решения для фотона (который есть локализованный в пространстве сгусток энергии электромагнитного поля), распространяющего со скоростью света только в одном (в отличие от электромагнитной волны) направлении.

DOI: 10.21883/JTF.2017.10.44988.2204

\section{Введение}

В 70-х годах прошлого века крупнейший физиктеоретик П.А.М. Дирак в лекциях по квантовой теории поля писал [1]:

„Необходимо, чтобы квантовая теория поля базировалась на таких понятиях и методах, которые можно было бы унифицировать с понятиями и методами остальной физики... Общепринятую трактовку квантовой теории поля следует рассматривать в качестве паллиатива без всякого будущего... Таким образом, квантовая теория поля на той стадии развития, на которой я ее оставляю, далека от завершения. Целый ряд направлений теории нуждается в дальнейшем развитии. Важнейшее из них найти какой-нибудь подход к вопросу о сильных взаимодействиях ${ }^{6}$.

В подтверждение этих высказываний знаменитого физика заметим, что в рамках современной теоретической физики структура основных элементов материи (электрона, протона, фотона и нейтрино) не только не рассчитана, но и не ясно каким образом записать уравнения, справедливые для описания структуры и физических процессов внутри объема элементарных частиц. Из этих цитат видим, что Дирак понимал недостатки квантовой теории поля и призывал к развитию новых методов описания физической реальности прежде всего сильных взаимодействий и, значит, структуры элементарных частиц. Несколько ранее (в 1960 г.) Л.Д. Ландау в своей последней опубликованной работе [2] писал, что “гамильтонов метод для сильных взаимодействий изжил себя и должен быть похоронен, конечно, со всеми почестями, которые он заслужил“. Так как структура свободного электрона не описывается ни уравнением Шрёдингера, ни релятивистскими уравнениями Дирака, то, значит, для описания структуры электрона, а так- же протона, фотона, нейтрино и других элементарных частиц нужна новая физика микромира, необходимость построения которой осознавалась многими выдающимися физиками XX века.

„Новая физическая теория, которую мы все так ждем - писал академик И.Е. Тамм - должна быть безупречно последовательной и логичной“ [3]. Но в современной теории используют аппарат, предполагающий точечный характер частиц [4]. Поэтому в такой теории возникают бесконечности. Отсюда и использование приема „перенормировки“, который Р. Фейнман называл „дурацким приемом“ [5], а П.А.М. Дирак бессмысленным с точки зрения математики. „В математике величину отбрасывают только в том случае, если она оказывается бесконечно малой, а не из-за того, что она бесконечно велика и от нее хотят избавиться!“ [6]. По мнению Дирака ,...основные уравнения неверны. Их нужно существенно изменить, с тем, чтобы в теории вообще не возникали бесконечности... Это условие потребует каких-то очень серьезных изменений“ [6]. В.К. Гейзенберг считал, что „развитие теоретической физики частиц с самого начала ведется неверно. Так как кварки не наблюдаемы и им приписываются большие массы, „представляется весьма затруднительной любая попытка придать сколько-нибудь определенный смысл утверждению „Протон состоит из трех кварков“", поскольку в этом случае утрачен определенный смысл и выражения „состоять из“ и слова „кварк“ [7].

В гамильтоновом методе учитываются силы взаимодействия между частицами. Часто эти силы являются определяющими, и тогда этот метод дает хорошие результаты, согласующиеся с измерениями. Но гамильтонов метод, который Ландау предлагал „похоронить“ [2], не применим для описания структуры отдельной изолированной частицы. Не применим потому, что в этом ме- 
тоде не учитываются внутренние силы, действующие на саму частицу внутри ее объема. В случае изолированной частицы эти внутренние силы, зависящие от плотности электрического заряда, тока и их пространственных и временных производных, компенсируют кулоновские силы отталкивания и именно поэтому возможно существование частицы в виде компактного сгустка электрического заряда, тока и создаваемого ими поля. Вне объема частицы внутренние силы отсутствуют, так как здесь плотность электрического заряда и тока равны нулю. Возникает вопрос: как построить корректную теорию, учитывающую эти внутренние силы?

Метод построения физической теории микромира, способной в принципе описать структуру электрона, протона и других элементарных частиц (а значит, и так называемые сильные взаимодействия), изложен в работах [8-14], в которых приведены и некоторые первые важные для практических приложений количественные результаты, согласующиеся с данными экспериментальных измерений. Метод основан на фундаментальных законах сохранения электрического заряда, энергии, потока энергии (импульса) и его момента. Цель этой работы в том, чтобы рассмотреть наиболее простой, но важный случай электромагнитного поля без зарядов и токов и показать основы новой физической теории, показать возможность описания фотона как частицеподобного компактного сгустка электромагнитного (ЭМ) поля, показать возможность существования ЭМ поля в разных состояниях.

Способность фотона от Солнца или далекой звезды к фотоэффекту и эффекту Комптона указывает на его компактность, на сохранение его характерных размеров в процессе распространения. Это важнейшая характеристика фотона. Так как компактный фотон, рожденный в малой области пространства, распространяется в каком-нибудь одном направлении, а волна во всех трех, фотон следует отличать от ЭМ волны. При этом уравнения состояния ЭМ поля фотона и волны (так называемые „материальные“ уравнения) должны быть разными: линейные для волны и нелинейные для фотона. Получим нелинейные уравнения для описания динамики ЭМ поля фотона и его структуры, исходя только из фундаментальных законов сохранения.

\section{Способ получения общих уравнений электродинамики, применимых и внутри объема элементарных частиц}

Задача фундаментальной физики не только в том, чтобы записать математические уравнения, описывающие динамику и структуру реальных объемных, неточечных частиц и соответствующих им полей, но также и в том, чтобы по возможности понять на основе нескольких фундаментальных постулатов, почему эти уравнения имеют именно такой вид. Прежде всего хотелось бы понять, почему уравнения Максвелла, описывающие динамику ЭМ поля, имеют именно такой хорошо всем известный вид. Покажем, каким образом это можно сделать, исходя из фундаментального закона сохранения электрического заряда с плотностью $\rho$ и плотностью тока $\mathbf{j}$, который в дифференциальной форме имеет вид

$$
\partial \rho / \partial t+\operatorname{div} \mathbf{j}=0 .
$$

Выразим плотность электрического заряда $\rho$ через некоторый вектор D так, что

$$
\rho=\operatorname{div} \mathbf{D} /(4 \pi) \rightarrow \operatorname{div} \mathbf{D}=4 \pi \rho .
$$

Это можно сделать многими способами, так как вектор D определен неоднозначно, с точностью до ротора произвольной функции rotF. Несмотря на эту неоднозначность для любого вектора $\mathbf{D}$, удовлетворяющего условию (2), поток вектора D через любую замкнутую поверхность, как хорошо известно из математики, всегда равен величине электрического заряда, находящегося внутри этой поверхности. Поэтому вектор D будем называть вектором индукции электрического заряда или просто электрической индукцией.

Далее, вычислим частную производную от левой и правой частей уравнения (2), тогда с учетом (1) получим: $\operatorname{div}(\partial \mathbf{D} / \partial t+4 \pi \mathbf{j})=0$. Формальное решение этого уравнения можно записать в виде

$$
4 \pi \mathbf{j}=c \operatorname{rot} \mathbf{H}-\frac{\partial \mathbf{D}}{\partial t},
$$

где $\mathbf{H}$ - пока произвольный вектор, $c$ - электродинамическая постоянная. Уравнения (2) и (3) - это первая пара уравнений Максвелла в среде (в единой теории поля среда - это поле и поле - это среда). Из (2) и (3) легко выразить $\rho$ и ј и поэтому эти уравнения можно рассматривать как полевое решение фундаментального уравнения (1), выраженное через векторы электрической индукции D и напряженности магнитного поля Н. Таким образом, конкретный вид этой пары уравнений Максвелла (2) и (3) обусловлен фундаментальным законом сохранения электрического заряда (1), который при этом для любых векторов D и Н выполняется всегда и автоматически.

Можно ли получить вторую пару уравнений Максвелла, исходя только из фундаментальных законов сохранения? Оказывается можно, причем также просто. С этой целью проведем анализ закона сохранения электрического заряда (1) иным способом. Введем оператор Даламбера $\square \equiv \Delta^{*}$, который на любую функцию действует по правилу

$$
\Delta^{*} n \equiv \Delta n-\frac{1}{c^{2}} \frac{\partial^{2} n}{\partial t^{2}}, \quad \Delta n \equiv \frac{\partial^{2} n}{\partial x^{2}}+\frac{\partial^{2} n}{\partial y^{2}}+\frac{\partial^{2} n}{\partial z^{2}} .
$$

Вычислим величины:

$$
n \equiv \rho / e, \quad \mathbf{u} \equiv \mathbf{j} / e, \quad \rho_{m} \equiv b \Delta^{*} n, \quad \mathbf{j}_{m} \equiv b \Delta^{*} \mathbf{u} .
$$

Здесь $e-$ постоянная, равная электрическому заряду протона, $b$ - постоянная, размерность которой для 
удобства выберем такой, чтобы размерности величин $\rho$ и $\rho_{m}$ совпадали бы. Это наше право и мы им воспользуемся. Теперь подействуем оператором Даламбера на уравнение (1) и, учитывая обозначения (4), получим

$$
\partial \rho_{m} / \partial t+\operatorname{div} \mathbf{j}_{m}=0
$$

Из (1) и (5) следует, что для произвольной изолированной физической системы (ИФС) постоянна не только интегральная (полная) величина электрического заряда $\langle\rho\rangle\langle\rho\rangle \equiv \iiint \rho d x d y d z$, но и еще одна величина $\left\langle\rho_{m}\right\rangle$, которую тоже будем называть зарядом. Учитывая, что на границе любой ИФС все физические величины (по определению ИФС) достаточно быстро убывают по величине и равны нулю, находим

$$
\begin{aligned}
\left\langle\rho_{m}\right\rangle & \equiv \iiint \rho_{m} d x d y d z=b\left\langle\Delta n-\frac{1}{c^{2} e} \frac{\partial^{2} n}{\partial t^{2}}\right\rangle \\
& =0-\frac{b}{c^{2} e} \frac{d^{2}}{d t^{2}}\langle\rho\rangle=0 .
\end{aligned}
$$

Видим, что интегральная величина нового заряда с плотностью $\rho_{m} \neq 0$ всегда для любой ИФС равна нулю. Поскольку, несмотря на тщательные поиски, магнитных монополей в природе не обнаружено (интегральные величины магнитных зарядов всех частиц равны нулю), эту величину условимся называть магнитным зарядом или $m$-зарядом, в отличие от обычного электрического $e$-заряда, а $\rho_{m}-$ плотностью $m$-заряда.

Далее, решение уравнения (5) для плотности магнитного заряда $\rho_{m}$ и плотности магнитного тока $\mathbf{j}_{m}$ можно записать так же, как и уравнения (1)-(3) [10], а именно

$$
\operatorname{div} \mathbf{B}=4 \pi \rho_{m}, \quad \frac{\partial \mathbf{B}}{\partial t}+c \operatorname{rot} \mathbf{E}=-4 \pi \mathbf{j}_{m},
$$

где $\mathbf{B}$ и $\mathbf{E}$ - произвольные векторы, которые условимся называть соответственно индукцией магнитного поля и напряженностью электрического поля. В пределе точечных частиц, когда их размерами можно пренебречь, вне их объема имеем $\rho=0, \mathbf{j}=0($ но $\langle\rho\rangle \neq 0,\langle\mathbf{j}\rangle \neq 0)$, $\rho_{m}=0, \mathbf{j}_{m}=0,\left\langle\rho_{m}\right\rangle=0,\left\langle\mathbf{j}_{m}\right\rangle=0$. При усреднении уравнений по объемам, гораздо большим объемов частиц, правые части в уравнениях (2), (3) не исчезают, а заменяются соответствующими дельта-функциями, потому что $\langle\rho\rangle \neq 0,\langle\mathbf{j}\rangle \neq 0$, тогда как правые части (7) исчезают: $\left\langle\rho_{m}\right\rangle=0,\left\langle\mathbf{j}_{m}\right\rangle=0$. При этом получаются уравнения Максвелла для точечных частиц, если их дополнить простыми уравнениями состояния („материальными“ уравнениями) для „вакуума“ в виде: $\mathbf{D}=\mathbf{E}, \mathbf{B}=\mathbf{H}$. В макроскопических процессах справедливы уравнения (7) с нулевыми правыми частями, которые играют важную роль только внутри объема частиц.

В уравнениях (2), (3), (7) векторы D, E, B, Н пока произвольные, но, забегая вперед, отметим, что они должны удовлетворять некоторым уравнениям связи, таким, чтобы выполнение фундаментального закона сохранения энергии было бы не только возможным, но и гарантированным. Оказывается, что для уравнений $(2)-(4)$, (7) это возможно [10], а вот если бы мы вместо (4) ввели новую сохраняющуюся величину с плотностью $\rho_{2}=\Delta^{*} \rho_{m}=\Delta^{*}\left(\Delta^{*} \rho\right)$ или тем более $\rho_{3}=\Delta^{*} \rho_{2}$, то в таком случае согласованное выполнение уравнений (2), (3), (7) и фундаментальных законов сохранения импульса и энергии стало бы невозможно и именно поэтому величины $\rho_{2}, \rho_{3}$ в отличие от $\rho_{m}$ не имеют физического смысла. Подчеркнем также, что если плотность магнитного заряда $\rho_{m}$ считать никак не связанной с плотностью электрического заряда $\rho$, то невозможно было бы построить самосогласованную теорию, в которой имело бы место одновременное выполнение системы уравнений (2), (3), (7) и фундаментальных законов сохранения импульса и энергии. При наличии связей (4) построение такой теории возможно [10].

Известно, что ЭМ поле в среде описывается четырьмя векторами D, E, В и Н. В рамках теории поля среда это поле и поле - это среда, поэтому в общем случае даже в „вакууме“ будем описывать ЭМ поле четырьмя векторами $\mathbf{D}, \mathbf{E}, \mathbf{B}$ и Н. Это формально согласуется с современными теориями нелинейной электродинамики вакуума (см., например, $[15,16])$. В некоторых из них отличия D от $\mathbf{E}$ и $\mathbf{B}$ от $\mathbf{H}$ малы и существенны только в очень сильных полях $\sim B_{q}=m^{2} c^{3} /(e \hbar)=4.41 \cdot 10^{13} \mathrm{G}$ $(m-$ масса электрона). Но в такой нелинейной электродинамике решений для фотона, рассматриваемого как компактный сгусток ЭМ поля (как ЭМ солитон), пока не найдено и, по-видимому, их не существует (см. ниже). В развиваемой в этой работе новой нелинейной электродинамики вакуума отличия $\mathbf{D}$ от $\mathbf{E}$ и $\mathbf{B}$ от $\mathbf{H}$ могут быть весьма существенны уже при малых полях, например в объеме фотона оптического спектра. Кроме того, ЭМ поле может существовать в разных состояниях, т.е. с разной связью между векторами $\mathbf{D}, \mathbf{E}, \mathbf{B}$ и $\mathbf{H}$ и разной зависимостью плотности энергии поля от векторов $\mathbf{D}$ и $\mathbf{B}$.

Заметим, что между введенным по формулам (4) $m$-зарядом с плотностью $\rho_{m} \neq 0,\left\langle\rho_{m}\right\rangle=0$ и кварками существует довольно много общего. И кварки, и $m$-заряд существуют только в объеме частиц. Они не могут быть удалены из объема частиц никакими силами и энергиями, они ответственны за целостность (компактность) частиц. Но нет никакой необходимости введения в теорию неточечных частиц [8-14] таких весьма странных гипотетических объектов, как кварки. В этой теории им просто нет места и они не решают никаких проблем, но создают новые. Поэтому проще считать, что в природе их нет.

\section{Роль закона сохранения энергии при построении нелинейной электродинамики фотона и неточечных частиц}

Электродинамика фотона описывается уравнениями (2), (3) и (7), в которых $\rho=0, \mathbf{j}=0, \rho_{m}=0, \mathbf{j}_{m}=0$, 
но связь полей $\mathbf{E}$ и $\mathbf{H}$ с векторами $\mathbf{D}$ и $\mathbf{B}$ не может быть линейной. Найдем эту нелинейную связь, исходя из закона сохранения энергии.

Для фотона, как и для любой изолированной физической системы, должен выполняться закон сохранения энергии с плотностью $W_{e}$ и плотностью потока энергии $\mathbf{Q}^{e}$ в виде

$$
\partial W_{e} / \partial t+\operatorname{div} \mathbf{Q}^{e}=0 .
$$

Пусть $W_{e}=W_{e}(\mathbf{D}, \mathbf{B})$ - пока произвольная скалярная дифференцируемая функция от двух векторных величин D и B. Тогда, выполняя в (8) дифференцирование по времени $t$ и используя уравнения (2) и (7) с учетом равенств $\rho=0, \mathbf{j}=0, \rho_{m}=0, \mathbf{j}_{m}=0$, получим

$$
\left(\frac{\partial W_{e}}{\partial \mathbf{D}}\right)_{\mathbf{B}} c \operatorname{rot} \mathbf{H}-\left(\frac{\partial W_{e}}{\partial \mathbf{B}}\right)_{\mathbf{D}} c \operatorname{rot} \mathbf{E}+\operatorname{div} \mathbf{Q}^{e}=0 .
$$

Это равенство должно выполняться для любых фотонов и любых их комбинаций всегда, в любых ситуациях. Из (9) следует, что первые два слагаемых должны всегда выражаться через дивергенцию некоторого вектора $\mathbf{Q}^{e}$. Это возможно в том случае, если в (9) множитель, стоящий перед $\operatorname{rot} \mathbf{H}$, будет пропорционален $\mathbf{E}$, a множитель, стоящий перед $\operatorname{rot} \mathbf{E}$ будет пропорционален Н с тем же коэффициентом пропорциональности. Так как вектор $\mathbf{Q}^{e}$ пока не определен, коэффициент пропорциональности мы можем выбрать любым (он зависит от используемых единиц), например $c / 4 \pi$. Тогда получим $[10,11]$

$$
\begin{array}{cc}
\mathbf{E}=4 \pi\left(\frac{\partial W_{e}}{\partial \mathbf{D}}\right)_{\mathbf{B}}, & \mathbf{H}=4 \pi\left(\frac{\partial W_{e}}{\partial \mathbf{B}}\right)_{\mathbf{D}}, \\
W_{e} \equiv W_{e}(\mathbf{D}, \mathbf{B}), & Q^{e} \equiv \frac{c}{4 \pi}[\mathbf{E} \mathbf{H}] .
\end{array}
$$

Вектор $\mathbf{Q}^{e}$ принято называть вектором Пойнтинга. Заметим, что формулы (10) согласуются с дифференциалом внутренней энергии $U$ в диэлектрической среде при постоянной плотности и энтропии: $d U=(\mathbf{E} d \mathbf{D}+\mathbf{H} d \mathbf{B}) /(4 \pi)$ [15]. Кроме того, уравнения состояния поля в виде, аналогичном (10), получены в [16] с термодинамической точки зрения. При этом $W_{e}$ следует считать той частью плотности свободной энергии, которая зависит от $\mathbf{D}$ и $\mathbf{B}$.

Чтобы лучше прояснить связь новых уравнений динамики ЭМ поля (2)-(7), (10) с уравнениями Максвелла вместо магнитной индукции $\mathbf{B}$ и электрического поля $\mathbf{E}$ введем новые величины $\mathbf{B}_{0}$ и $\mathbf{E}_{0}$ по формулам

$$
\mathbf{B} \equiv \mathbf{B}_{0}+4 \pi b\left(\nabla n+\frac{1}{c^{2}} \frac{\partial \mathbf{u}}{\partial t}\right), \quad \mathbf{E} \equiv \mathbf{E}_{0}+\frac{4 \pi b}{c} \operatorname{rot} \mathbf{u} .
$$

Тогда, подставляя (12) в (7), с учетом (4) и (1) получим

$$
\frac{\partial \mathbf{B}_{0}}{\partial t}=-c \operatorname{rot} \mathbf{E}_{0}, \quad \operatorname{div} \mathbf{B}_{0}=0
$$

Видим, что (13) совпадают с соответствующими уравнениями Максвелла! Таким образом, учет магнитных зарядов и токов с плотностями, определяемыми формулами (4), приводит к появлению новых (по сравнению с теорией Максвелла) простых слагаемых в выражении для магнитной индукции В и напряженности электрического поля $\mathbf{E}$ (см. (12)). Эти слагаемые выражаются через первые производные от плотности электрического заряда и тока, и поэтому они важны при исследовании внутриатомных и ядерных физических процессов, где эти величины отличны от нуля и велики. Вне объема ядер частиц имеем $n \cong 0, \mathbf{u} \cong 0$ и, значит, $\mathbf{B} \cong \mathbf{B}_{0}$, $\mathbf{E} \cong \mathbf{E}_{0}$.

Векторы $\mathbf{B}_{0}$ и $\mathbf{E}_{0}$ можно выразить через потенциалы $\mathbf{A}$ и $\varphi$ по формулам

$$
\mathbf{B}_{0}=\operatorname{rot} \mathbf{A}, \quad \mathbf{E}_{0}=-\nabla \varphi-\frac{1}{c} \frac{\partial \mathbf{A}}{\partial t} .
$$

Тогда векторы В и $\mathbf{E}$ можно записать в симметричном виде $[10,11]$

$$
\begin{aligned}
& \mathbf{B}=\operatorname{rot} \mathbf{A}+4 \pi b\left(\nabla n+\frac{1}{c^{2}} \frac{\partial \mathbf{u}}{\partial t}\right), \\
& \mathbf{E}=\frac{4 \pi b}{c} \operatorname{rot} \mathbf{u}-\left(\nabla \varphi+\frac{1}{c} \frac{\partial \mathbf{A}}{\partial t}\right) .
\end{aligned}
$$

Нетрудно проверить, что из системы уравнений (2), (3), (7), (10) в случае $\rho=0, \mathbf{j}=0, \rho_{m}=0, \mathbf{j}_{m}=0$ следует закон сохранения импульса для фотонов в виде

$$
\begin{gathered}
\frac{\partial U_{k}^{e}}{\partial t}+\frac{\partial T_{i k}^{e}}{\partial x_{i}}=0, \quad \mathbf{U}^{e} \equiv \frac{[\mathbf{D} \mathbf{B}]}{4 \pi c}, \\
T_{i k}^{e} \equiv \frac{\left\{\delta_{i k}((\mathbf{D ~ E})+(\mathbf{B} \mathbf{H}))-D_{i} E_{k}-B_{i} H_{k}\right\}}{4 \pi}-W_{e} \delta_{i k} .
\end{gathered}
$$

Здесь вектор $\mathbf{U}^{e}-$ плотность импульса в классической электродинамике, а $T_{i k}^{e}-$ тензор натяжений ЭМ поля. Для любой скалярной функции $W_{e}=$ $=W_{e}(\mathbf{D}, \mathbf{B})=W_{e}\left(D^{2}, B^{2},(\mathbf{D} \mathbf{B})\right)$ легко убедиться, что тензор $T_{i k}^{e}=T_{k i}^{e}-$ симметричный. Значит, справедлив закон сохранения момента импульса с плотностью $\mathbf{M}^{e}=\left[\mathbf{R} \mathbf{U}^{e}\right], \mathbf{R} \equiv(x, y, z)=\left(x_{1} x_{2} x_{3}\right)$.

$\mathrm{B}$ классической электродинамике с $W_{e}=\left(\mathbf{D}^{2}+\mathbf{B}^{2}\right) /$ $(8 \pi)$ между плотностью потока энергии $\mathbf{Q}^{e}$ и плотностью импульса $\mathbf{U}^{e}$ в вакууме существует простая связь: $\mathbf{Q}^{e}=c^{2} \mathbf{U}^{e}$ или $[\mathbf{E} \mathbf{H}]=[\mathbf{D} \mathbf{B}]$. В современной теории нелинейной электродинамики вакуума $[17,18]$ вместо (10) принято использовать несколько иные (заметим, почти всегда эквивалентные) уравнения связи, а именно

$$
\begin{gathered}
\mathbf{D}=4 \pi(\partial L / \partial \mathbf{E})_{\mathbf{B}}, \quad \mathbf{H}=-4 \pi(\partial L / \partial \mathbf{B})_{\mathbf{E}}, \\
L=L\left(\mathbf{E}^{2}-\mathbf{B}^{2},(\mathbf{E B})\right),
\end{gathered}
$$

в которых векторы D и Н выражаются через векторы $\mathbf{E}$ и $\mathbf{B}$ с помощью функции Лагранжа $L$ для ЭМ поля. В общем случае $L-$ некоторая функция двух скалярных величин, которые, заметим, являются инвариантами преобразований Лоренца для ЭМ поля, а именно $\mathbf{E}^{2}-\mathbf{B}^{2}$ и $(\mathbf{E} \mathbf{B})$. Из (16) легко убедиться, что 
для произвольной дифференцируемой функции $L(16)$ справедливо следующее векторное уравнение:

$$
[\mathbf{E} \mathbf{H}]=[\mathbf{D} \mathbf{B}] \text {. }
$$

Это важное векторное равенство, являющееся следствием уравнений (16) современной теории нелинейной электродинамики вакуума $[17,18]$, справедливо во всех случаях, для произвольных функций Лагранжа $L$ для ЭМ поля и даже в тех некоторых случаях, когда (см. ниже) функции Лагранжа формально не существует.

В теории неточечных частиц [10], исходя из условия существования закона сохранения импульса любой изолированной физической системы, показано, что плотность потока энергии $\mathbf{Q}$ отличается от плотности импульса $\mathbf{U}$ всегда только постоянным множителем $c^{2}$. Иначе говоря, закон сохранения импульса и закон сохранения потока энергии (в общем, релятивистском случае) - это один и тот же физический закон. Поэтому простые связи $\mathbf{Q}=c^{2} \mathbf{U}$ и векторное равенство (17) справедливы всегда. Покажем, что равенство (17) позволяет нам найти конкретные виды возможных функций $W_{e}=W_{e}(\mathbf{D}, \mathbf{B})=W_{e}\left(D^{2}, B^{2},(\mathbf{D} \mathbf{B})\right)$.

Введем скалярные величины: $Z_{1} \equiv D^{2}, Z_{2} \equiv B_{2}, Z_{3} \equiv$ $\equiv(\mathbf{D} \mathbf{B})$. Тогда, исходя из условия $[\mathbf{E} \mathbf{H}]=[\mathbf{D} \mathbf{B}]$, для скалярной функции $F \equiv 4 \pi W_{e}=F\left(Z_{1}, Z_{2}, Z_{3}\right)$ получим уравнение

$$
W_{e}=\frac{F}{4 \pi}, \quad 4 \frac{\partial F}{\partial Z_{1}} \frac{\partial F}{\partial Z_{2}}-\left(\frac{\partial F}{\partial Z_{3}}\right)^{2}=1 .
$$

Граничные условия для этого уравнения: $F\left(Z_{1}=0, Z_{2}=\right.$ $\left.=0, Z_{3}=0\right)=0$ и $F<\infty$ при $Z_{i}<\infty, i=1,2,3$.

Анализ некоторого класса решений уравнения (18) проведен в работе [10]. Здесь мы приведем только шесть наиболее простых решений этого уравнения, в справедливости которых просто убедиться подстановкой в (18):

$$
\begin{gathered}
F_{1}=\left(D^{2}+B^{2}\right) / 2 \\
F_{2}=\frac{1}{2} \sqrt{\left(\left(D^{2} p+B^{2} / p\right)^{2}-4(\mathbf{D ~ B})^{2}\right)} \\
F_{3}=\sqrt{D^{2} B^{2}-(\mathbf{D ~ B})^{2}} \\
F_{4}=D B \operatorname{ch}(p) \pm(\mathbf{D ~ B}) \operatorname{sh}(p), \\
F_{5}=\sqrt{\left(\left(D^{2}+p\right)\left(B^{2}+p\right)\right)}-\bmod (p), \quad p=\mathrm{const} \\
F_{6}=\sqrt{\left(\left(D^{2}+B\right)\left(B^{2}+p\right)-(\mathbf{D} \mathbf{B})^{2}\right)}-\sqrt{b p}, \\
b=\mathrm{const}, \quad b p>0 .
\end{gathered}
$$

Решение $F_{1}$ соответствует ЭМ волне, для которой верны простейшие связи $\mathbf{D}=\mathbf{E}, \mathbf{H}=\mathbf{B}$ и поэтому уравнения Максвелла в вакууме сводятся к волновому уравнению. Решение $F_{2}$, возможно, соответствует электрону (?), $F_{3}-$ фотону, $F_{4}-$ нейтрино (?), $F_{5}$ или $F_{6}$ (при $b \neq 0, p \neq 0)$ - протону (?). Решения (19) впервые получены в работе [9], а (20) в [10].
Интересно, что с помощью (10) выражения для $F_{1}-F_{4}$ $\left(\right.$ и $\left.W_{e}\right)$ можно записать в виде одной формулы (это нетрудно проверить простыми вычислениями)

$$
W_{e n} \equiv \frac{\left(\mathbf{E}_{n} \mathbf{D}\right)+\left(\mathbf{H}_{n} \mathbf{B}\right)}{8 \pi}, \quad n=1,2,3,4,
$$

которая совпадает с выражением для энергии ЭМ поля в среде в классической электродинамике.

\section{Примеры конкретных аналитических структур фотонов}

Полная энергия фотона $\left\langle W_{e}\right\rangle$ связана с модулем его импульса $\left\langle U^{e}\right\rangle$ формулой $\left\langle W_{e}\right\rangle=c\left\langle U^{e}\right\rangle$. Для решения $F_{3}$ справедлива формула $W_{e}=c U^{e}$ в каждой точке пространства, поэтому состояние ЭМ поля в виде $F_{3}-$ соответствует, по-видимому, фотону - компактному в пространстве сгустку ЭМ поля. В этом случае структура и динамика фотона в предположении $\rho=0, \mathbf{j}=0$, $\rho_{m}=0, \mathbf{j}_{m}=0$ описываются следующей системой нелинейных уравнений:

$$
\begin{aligned}
& \mathbf{E}=[\mathbf{B} \mathbf{e}], \quad \mathbf{H}=[\mathbf{e} \mathbf{D}], \quad \mathbf{e} \equiv[\mathbf{D} \mathbf{B}] / w, \quad w \equiv \bmod [\mathbf{D} \mathbf{B}], \\
& \frac{\partial \mathbf{D}}{\partial t}=c \operatorname{rot} \mathbf{H}, \quad \operatorname{div} \mathbf{D}=0, \quad \frac{\partial \mathbf{B}}{\partial t}=-c \operatorname{rot} \mathbf{E}, \quad \operatorname{div} \mathbf{B}=0 .
\end{aligned}
$$

Здесь е - единичный вектор вдоль направления плотности импульса фотона. Из (19)-20) нетрудно получить законы сохранения энергии и импульса в виде (8) и (16). Граничные условия в каждый момент времени $t$ можно записать в виде $\mathbf{D}(t, \mathbf{R} \rightarrow \infty) \rightarrow 0$, $\mathbf{B}(t, \mathbf{R} \rightarrow \infty) \rightarrow 0, \mathbf{D}<\infty, \mathbf{B}<\infty$, полная энергии фотона $\left\langle W_{e}\right\rangle=\left\langle F_{3} / 4 \pi\right\rangle-$ конечная величина.

Заметим, что в работах $[17,18]$ для описания динамики ЭМ поля в вакууме тоже используются те же дифференциальные уравнения (22), в которых векторы D и Н выражаются через векторы $\mathbf{E}$ и В по формулам (16) с помощью функции Лагранжа $L$ для ЭМ поля. Важно обратить внимание на то, что уравнения связи полей в виде (10) и (16) не всегда являются эквивалентными. Чтобы убедиться в этом, легко проверить, что функциям $F_{1}$ и $F_{2}(19), F_{5}$ и $F_{6}(20)$ соответствуют функции Лагранжа:

$$
\begin{gathered}
L_{1}^{*}=\left(\mathbf{E}^{2}-\mathbf{B}^{2}\right) / 2, \\
L_{2}^{*}=\left(\left(\left(\mathbf{E}^{2}-\mathbf{B}^{2}\right) / 2 p\right)^{2}+(\mathbf{E} \mathbf{B})^{2} / p^{2}\right)^{1 / 2}, \\
L_{5}^{*}=\bmod (p)-\left(p\left(p-\mathbf{E}^{2}+\mathbf{B}^{2}\right)\right)^{1 / 2}, \\
L_{k}^{*} \equiv 4 \pi L_{k}, \quad k=1,2, \ldots, \\
L_{6}^{*}=(b p)^{1 / 2}-\left(b\left(p\left(p-\mathbf{E}^{2}+\mathbf{B}^{2}\right)-(\mathbf{E} \mathbf{B})^{2}\right) / p\right)^{1 / 2} .
\end{gathered}
$$

Но в случае $F_{3}(20)$ имеем $(\mathbf{E} \mathbf{B})=0$ и $\mathbf{E}^{2}-\mathbf{B}^{2}=0$ (см. (21)). Поэтому это особый случай, для которого формально $L_{3}(0,0)=0$, т.е. функции Лагранжа не 
существует, поскольку ее аргументы не изменяются и всегда равны нулю. Для решения $F_{4}(20)$ с учетом (10) имеет место равенство $\mathbf{E}^{2}-\mathbf{B}^{2}= \pm 2 \operatorname{sh}(p)(\mathbf{E} \mathbf{B})$, т.е. переменные $\mathbf{E}^{2}-\mathbf{B}^{2}$ и $(\mathbf{E} \mathbf{B})$ у функции Лагранжа не являются независимыми. Это тоже особый случай. В обоих этих случаях из уравнений (10) невозможно выразить вектор D через $\mathbf{E}$ и $\mathbf{B}$ и поэтому для заданных функций $F_{3}$ и $F_{4}$ нельзя построить соответствующие функции Лагранжа $L_{3}$ и $L_{4}$. Поэтому уравнения связи полей $\mathbf{D}, \mathbf{E}, \mathbf{B}$ и $\mathbf{H}$ в виде (10) являются более общими, чем уравнения связи (16). Заметим, что имеет место следующая связь между $W_{e}$ и $L$ :

$$
W_{e}(\mathbf{D}, \mathbf{B})=\frac{(\mathbf{E} \mathbf{D})}{4 \pi}-L(\mathbf{E}, \mathbf{B})
$$

Отсюда видно, что для функций $F_{3}$ и $F_{4}$ имеем $L_{3}=0$ и $L_{4}=0$.

При $b=p=1 / a^{2}$ функция $L_{6}$ совпадает с функцией Лагранжа нелинейной электродинамики БорнаИнфельда [19], которая, согласно [18], является выделенной среди всех нелинейных электродинамик. В пределе $b=p \rightarrow+0$ имеем $F_{6} \rightarrow F_{3}$. В этом же пределе действительные значения $L_{6} \rightarrow 0$ только при $(\mathbf{E} \mathbf{B})=0$ и $\mathbf{E}^{2}-\mathbf{B}^{2}=0$, что согласуется с (21).

В общем случае система уравнений (21), (22) является нелинейной, но может стать и линейной для тех частных решений, в которых реализуется постоянство в пространстве и во времени единичного вектора е, т.е. когда, например, $\mathbf{e}=(0,0,1)$. Но заранее неочевидно, что такие решения существуют. Поэтому можно предположить, что такие решения есть, найти их и затем тщательно проверить, что все уравнения и наше предположение о постоянстве единичного вектора $\mathbf{e}=(0,0,1)$ действительно выполняются в каждой точке пространства и в любой момент времени.

Если $\mathbf{e}=(0,0,1)$, то уравнения (22) с учетом (21) можно записать в виде

$$
\begin{gathered}
\frac{\partial \mathbf{D}}{\partial t}+c \frac{\partial \mathbf{D}}{\partial z}=0, \quad \operatorname{div} \mathbf{D}=0, \quad \mathbf{D}=\left(D_{x}, D_{y}, 0\right), \\
\frac{\partial \mathbf{B}}{\partial t}+c \frac{\partial \mathbf{B}}{\partial z}=0, \quad \operatorname{div} \mathbf{B}=0, \quad \mathbf{B}=\left(B_{x}, B_{y}, 0\right) .
\end{gathered}
$$

Общее решение нестационарных векторных уравнений системы (24) имеет простой вид

$$
\mathbf{D}=\mathbf{D}(x, y, z-c t), \quad \mathbf{B}=\mathbf{B}(x, y, z-c t),
$$

где зависимость от переменных $x, y, z-c t$ может быть произвольной. Остальные два скалярные уравнения тоже имеют простое решение в виде

$$
\mathbf{D}=[\nabla G \mathbf{e}], \quad \mathbf{B}=[\nabla F \mathbf{e}], \quad \mathbf{e}=(0,0,1),
$$

где $G$ и $F-$ произвольные дифференцируемые функции от трех независимых переменных $x, y, z-c t$, конкретный вид которых должен быть выбран таким, чтобы вектор е, определенный формулой (21), в каждой точке пространства в любой момент времени был бы равен $\mathbf{e}=(0,0,+1)$. Это не простое условие, но удовлетворить ему можно.

Одно из простых решений фотонного типа системы уравнений (21), (22) с учетом полученных формул (24)-(26) можно записать в виде [11]

$$
\begin{gathered}
B_{x}=\frac{A\left(R^{2}-y^{2}\right) f}{\lambda R^{3}}, \quad B_{y}=\frac{A x y f}{\lambda R^{3}}=-D_{x}, \\
D_{y}=-\frac{A\left(R^{2}-x^{2}\right) f}{\lambda R^{3}}, \\
B_{z}=0=D_{z} \rightarrow \mathbf{e}=(0,0,1), \\
R=\left(x^{2}+y^{2}+g^{2}\left((z-c t)^{2}+a^{2} \lambda^{2}\right)\right)^{1 / 2}, \\
f=f((z-c t) / \lambda)=\sin (k(z-c t)) /\left(d^{2}+((z-c t) / \lambda)^{2}\right)^{s}, \\
k=2 \pi / \lambda ; \\
W_{e}(a=d, s=1 / 2)=\frac{A^{2} g^{2} \sin ^{2}(k(z-c t))}{4 \pi R^{4}} \geq 0, \\
\left\langle W_{e}\right\rangle=\frac{\pi A^{2}}{8 a \lambda}(1-\exp (-4 \pi a)) .
\end{gathered}
$$

Здесь $\lambda-$ характерный продольный размер фотона (длина волны), $A, a, d, g, s-$ постоянные. $E_{x}=B_{y}$, $E_{y}=-B_{x}, E_{z}=0, H_{x}=-D_{y}, H_{y}=D_{x}, H_{z}=0$. Полная энергия фотона обратно пропорциональна длине волны $\lambda$ (если постоянные $A, a$ и $d>0$ не зависят от $\lambda$ ). Если считать, что для элементарного фотона его энергия $\left\langle W_{e}\right\rangle=h c / \lambda$, где $h-$ постоянная Планка, то из (24) легко выразить $A$ через $a$ и $h$. Из (27) и (28) видно, что при $a=d \sim 1$ параметр $g$ - это отношение поперечного размера фотона к продольному $\lambda$. В рассматриваемом случае полная энергия фотона $\left\langle W_{e}\right\rangle$ от $g$ не зависит. Если предположить, что поперечные размеры фотона, излучаемого или поглощаемого атомом водорода, порядка размера атома $a_{0} \sim 5 \cdot 10^{-9} \mathrm{~cm}$, то, приравнивая энергию фотона $h c / \lambda$ энергии ионизации атома водорода $e^{2} /\left(2 a_{0}\right)$, получим $\lambda=4 \pi a_{0} / \alpha, g \sim a_{0} / \lambda=\alpha /(4 \pi)$, где $\alpha \approx 1 / 137$ - постоянная тонкой структуры. В точном решении (27), (28) и ниже функция $f(z-c t)$ может быть произвольной от своего аргумента, но такой, чтобы величины $W_{e}$ и $\left\langle W_{e}\right\rangle$ были бы конечными.

Приведем еще одно точное аналитическое решение системы уравнений (21), (22), описывающее структуру фотона - компактного сгустка ЭМ поля, распространяющегося в пространстве строго в одном направлении и сохраняющего при этом свою структуру. В этом втором решении единичный вектор е тоже постоянен и равен $\mathbf{e}=(0,0,1)$. Значит, тоже справедливы формулы

$$
\begin{aligned}
B_{z}=0=D_{z} \rightarrow w & =\sqrt{\mathbf{D}^{2} \mathbf{B}^{2}-(\mathbf{D} \mathbf{B})^{2}} \\
& =\sqrt{\left(D_{x} B_{y}-D_{y} B_{x}\right)^{2}},
\end{aligned}
$$

из которых следует, что компонента вектора $e_{z}=$ $=\left(D_{x} B_{y}-D_{y} B_{x}\right) / w($ см. (21)) для любых функций $G$ 
и $F$, входящих в (26), может принимать только два значения $e_{z}= \pm 1$. Значит, функции $G$ и $F$ надо выбрать такими, чтобы в любой точке пространства с координатами $x, y, z$ и в любой момент времени $t$ параметр $P \equiv D_{x} B_{y}-D_{y} B_{x}$ имел бы одинаковый знак, например, был бы положительным (точнее, не отрицательным). Если для некоторых выбранных нами функций $G$ и $F$ в соседних точках пространства величина $P$ имеет разные знаки, то тогда между этими точками величина $e_{z}$ не является непрерывной функцией и испытывает скачок от $e_{z}=-1$ до $e_{z}=+1$. Но это недопустимо по условию о предполагаемом виде решения исходной нелинейной системы уравнений (21), (22), в рамках которого получены решения этих уравнений в виде $(24)-(26)$. Поэтому такие функции не могут быть искомыми решениями и должны быть заменены на другие. Оказывается, что подобрать две произвольные функции $G(x, y, z-c t)$ и $F(x, y, z-c t)$, для которых выполняется условие

$$
P=D_{x} B_{y}-D_{y} B_{x}=-\frac{\partial G}{\partial y} \frac{\partial F}{\partial x}+\frac{\partial G}{\partial x} \frac{\partial F}{\partial y} \geq 0
$$

в любой точке пространства и во все моменты времени, не такая уж простая задача, как может показаться на первый взгляд. Чтобы в этом убедиться читателю рекомендуется попробовать найти самостоятельно две такие функции.

Решению (27), (28) соответствуют функции

$$
G=\frac{A x f}{\lambda R}, \quad F=\frac{A y f}{\lambda R} .
$$

Легко проверить, что если мы вместо (31) запишем

$$
\begin{gathered}
G=Q\left(q_{x}\right) f(z-c t), \quad F=S\left(q_{y}\right) f(z-c t), \\
q_{x}=\frac{x}{R}, \quad q_{y}=\frac{y}{R}, \quad \frac{\partial Q}{\partial q_{x}} \frac{\partial S}{\partial q_{y}} \geq 0, \\
R=\left(x^{2}+y^{2}+g^{2}(z-c t)^{2}+b^{2}\right)^{1 / 2},
\end{gathered}
$$

(где $b$ и $g$ - постоянные, $Q\left(q_{x}\right)$ и $S\left(q_{y}\right)$ - произвольные функции, но такие, что их производные по своим аргументам имеют одинаковые знаки при любых допустимых значениях $q_{x}$ и $\left.q_{y}, \bmod \left(q_{x}\right)<1, \bmod \left(q_{y}\right)<1\right)$, то формулы (21), (26), (32) тоже будут решением исходной системы уравнений $(21)-(22)$ и описывать фотон, т.е. некоторый частицеподобный сгусток ЭМ поля, распространяющийся вдоль оси $z$ со скоростью $c$. Отметим, что подобрать функции $Q\left(q_{x}\right)$ и $S\left(q_{y}\right)$, удовлетворяющие неравенству (32) для всех возможных $q_{x}$ и $q_{y}$, несложно, например, в виде полиномов или в виде

$$
Q\left(q_{x}\right)=\operatorname{tg}\left(s q_{x}\right), \quad S\left(q_{y}\right)=\operatorname{tg}\left(s q_{y}\right), \quad s=\text { const }<\pi / 2,
$$

Функции вида

$$
\begin{gathered}
G=\frac{x V(z-c t)}{r}, F=\frac{y V(z-c t)}{r}, \\
r=\left(P^{4}+2 b p^{2}+a\right)^{1 / 4}, P^{2}=x^{2}+y^{2}+g^{2}(z-c t)^{2}
\end{gathered}
$$

(где $V(z-c t)$ - произвольная непрерывная, дифференцируемая, ограниченная функция от одной переменной $z-c t, a, b, g-$ положительные постоянные) тоже являются точными аналитическими решениями исходной нелинейной системы уравнений (21), (22), для которых условие (30) выполняется во всем пространстве, так как

$$
w=P=\frac{V^{2}}{r^{6}}\left(\left(P^{2}+b\right)\left(P^{2}-x^{2}-y^{2}\right)+b P^{2}+a\right) \geq 0 .
$$

Из (35) легко видеть, что полная энергия фотона $\langle W\rangle$, пропорциональная интегралу по всему пространству от величины $w$, величина конечная: $\langle W\rangle=\langle w\rangle /(4 \pi)<\infty$.

Видим, что структура фотона как частицеподобного сгустка ЭМ поля, распространяющегося в вакууме со скоростью $c$ вдоль какого-то одного направления и сохраняющего при этом свою форму, может быть весьма многообразной. В частности, в лазере испущенные отдельными атомами фотоны могут следовать друг за другом и образовывать длинные цуги колебаний поля, которым соответствуют в формуле (27) параметр $d \gg 1$.

\section{Обоснование существования разных состояний ЭМ поля}

Ключевую идею этой работы можно выразить следующим образом. Эффект Комптона и фотоэффект указывают на то, что фотон - это компактный, подобный частице трехмерный сгусток энергии ЭМ поля. Важно, что этот сгусток распространяется в отличие от электромагнитной волны только в одном направлении и поэтому сохраняет свою структуру и максимальную амплитуду поля при удалении от места своего рождения. Уравнения Максвелла в вакууме со связями $\mathbf{D}=\mathbf{E}, \mathbf{H}=\mathbf{B}$ сводятся к волновому уравнению $\square \mathbf{E}=0$ и поэтому хорошо описывают электромагнитные волны, распространяющиеся во всех направлениях от источника, но эти уравнения не описывают фотон как компактный, подобный частице сгусток ЭМ поля. Так как и ЭМ волны, и фотоны это ЭМ поля, мы вынуждены считать, что эти поля в чем-то разные, находящиеся в разных состояниях и поэтому описываемые разными уравнениями связи между векторами D, E, Н и В. Для волн имеем простые линейные связи $\mathbf{E}=\mathbf{D}, \mathbf{H}=\mathbf{B}$, а для фотонов и частиц связи (21) нелинейные с $\mathbf{E} \neq \mathbf{D}, \mathbf{H} \neq \mathbf{B}$ даже в вакууме.

Простая и важная задача о структуре фотона в вакууме до сих пор не решена, потому что уравнения Максвелла с $\mathbf{E}=\mathbf{D}, \mathbf{H}=\mathbf{B}, \rho=0, \mathbf{j}=0$ не имеют решений фотонного типа вида $\mathbf{E}=\mathbf{F}(x, y, z-V t), V=$ const, ограниченных во всем пространстве с конечной полной энергией $\left\langle W_{e}\right\rangle<\infty$. Это легко доказать [20] для трех случаев: $V=c, V<c, V>c$. В общем случае $\mathbf{E}(t, x, y, z)$ решение уравнений Максвелла (волнового уравнения $\square \mathbf{E}=0$ ) представляет собой расходящийся в разных направлениях поток энергии волн [20], в то время как у фотона поток энергии направлен в одном направлении и не расходится в пространстве. Поэтому состояния электромагнитного поля в виде ЭМ волны и в виде фотона - это разные состояния поля. 


\section{Замечания}

1. Взаимодействие фотона $\left(F_{3}\right)$ с ЭМ волной $\left(F_{1}\right)$ в вакууме отсутствует, т.е. поля волны $\mathbf{E}_{0}=\mathbf{D}_{0}, \mathbf{H}_{0}=\mathbf{B}_{0}$ с плотностью энергии $W_{0}=\left(D_{0}^{2}+B_{0}^{2}\right) / 8 \pi$ не влияют на распространение фотона, для которого $\mathbf{E} \neq \mathbf{D}, \mathbf{H} \neq \mathbf{B}$. Но взаимодействие фотонов с фотонами, описываемое нелинейными уравнениями (21), (22), должно быть и оно наблюдалось в экспериментах [21] по рассеянию лазерных фотонов на гамма квантах. Факт существования такого рассеяния в вакууме доказывает нелинейность электродинамики фотонов.

2. Существующий в природе дуализм волна-частица есть простое и естественное следствие того, что фотоны, электроны и другие неточечные частицы представляют собой компактные сгустки поля и энергии. При этом сгусток проявляет себя (при определенных условиях) как частица, а поле конечного объема - как волна.

3. ЭМ поле может находиться в разных состояниях. В частности, для ЭМ поля в состоянии фотона, описываемого системой нелинейных уравнений (21), (22), имеем $\mathbf{D} \neq \mathbf{E}$ и $\mathbf{B} \neq \mathbf{H}$ даже в слабых полях светового фотона. Вывод о существовании в природе различных состояний ЭМ поля чрезвычайно важен для будущего развития физики, так как открываются огромные возможности для корректного описания удивительно богатого многообразия наблюдаемых ЭМ явлений (в частности, разных типов шаровых молний), которое невозможно описать в рамках ныне существующей слишком упрощенной теории электродинамики на основе уравнений Максвелла с необоснованным и примитивным предположением, что в вакууме возможны только простейшие связи полей в виде: $\mathbf{D}=\mathbf{E}$ и $\mathbf{B}=\mathbf{H}$. Такое состояние ЭМ поля в вакууме действительно реализуется в природе и соответствует ЭМ волнам, но у нас нет оснований считать, что оно единственно возможное. Более того, известная способность фотона, излученного Солнцем или далекой звездой, вызывать фотоэффект и эффект Комптона в земных условиях однозначно указывает на то, что фотон - это компактный сгусток ЭМ поля, в котором плотность энергии поля сохраняется внутри его объема, перемещающегося в пространстве со скоростью света в одном выделенном направлении. Это кардинально отличается от ЭМ волны, которая распространяется от области ее генерации во всех направлениях. Поэтому состояния ЭМ поля в виде фотона и в виде ЭМ волны - это разные состояния ЭМ поля, описываемые уравнениями связи векторов $\mathbf{D}, \mathbf{E}, \mathbf{B}$ и $\mathbf{H}$ в виде (10) с разными функциями $W_{e}(\mathbf{D}, \mathbf{B})\left(\right.$ см. $F_{3}(20)$ и $F_{1}(19)$ соответственно).

4. Теория точечных частиц проста и удобна при решении известного узкого круга задач. Но она не применима даже в принципе для расчета логически корректным способом структуры реальных неточечных частиц, так как слишком упрощена. Поскольку основные свойства материи определяются, очевидно, физическими процессами на масштабах, сравнимых с реальными размерами частиц, отсюда следует необходимость построения логически безупречной физической теории неточечных частиц, лишенной проблем с бесконечностями. Основы такой теории с учетом отличной от нуля плотности электрического заряда $\rho$ и тока ј построены в [8-10], а в этой работе для частного случая $\rho=0, \mathbf{j}=0$ очень простым способом на основе только фундаментальных законов сохранения получен ряд полезных результатов.

\section{Заключение}

1. На примере фотона простым способом показано, что существует метод построения новой электродинамики поля в вакууме только на основе гипотезы существования в природе фундаментальных законов сохранения электрического заряда, энергии, потока энергии (импульса) и его момента. Применение этого метода к протону, электрону и другим заряженным частицам тоже возможно и выполнено подробно в работах [8-10], а также приведено без доказательства в работах $[11,12,14]$. В этом случае решение проблемы гораздо сложнее, так как требуется записать уравнение для электрического тока. При этом мы получим уравнения, применимые и внутри объема электрона, протона и других частиц, и, таким образом, найдем тот „какой-нибудь подход к вопросу о сильных взаимодействиях“, к поиску которого призывал П.А.М. Дирак [1],

2. С учетом того, что структура фотона, понимаемого как частицеподобный сгусток энергии ЭМ поля, не описывает система уравнений Максвелла в вакууме с $\mathbf{D}=\mathbf{E}$ и $\mathbf{B}=\mathbf{H}$, но естественным образом описывается новой электродинамикой вакуума с $\mathbf{D} \neq \mathbf{E}$ и $\mathbf{B} \neq \mathbf{H}$ (см. (21)), мы приходим к важному выводу, а именно ЭМ поле может находиться в разных состояниях, с разными уравнениями состояния. Отсутствие в системе уравнений Максвелла в вакууме со связями $\mathbf{E}=\mathbf{D}, \mathbf{H}=\mathbf{B}$ частицеподобных решений фотонного типа показывает, что эти уравнения не являются универсальными, что существуют ЭМ процессы, которые они не описывают. Реальная нелинейная электродинамика вакуума несравненно разнообразнее, богаче, чем примитивная линейная электродинамика со связями $\mathbf{D}=\mathbf{E}$ и $\mathbf{B}=\mathbf{H}$.

3. Уравнения нелинейной электродинамики получены только из фундаментальных законов сохранения электрического заряда, энергии, импульса и момента импульса. В асимптотическом пределе больших по сравнению с размерами частиц расстояний (или что то же самое в предельном случае точечных частиц), когда $W_{e} \approx\left(\mathbf{D}^{2}+\mathbf{B}^{2}\right) /(8 \pi)$, уравнения нелинейной электродинамики совпадают с уравнениями Максвелла.

4. Получены точные частные решения нелинейных уравнений (21), (22) динамики ЭМ поля без зарядов и токов для компактных сгустков поля - фотонов, которые переносят энергию ограниченного в пространстве поля со скоростью света. Подобных решений уравнений Максвелла для случая $\mathbf{E}=\mathbf{D}, \mathbf{H}=\mathbf{B}$ нет, поэтому этот частный случай не является универсальным даже для вакуума $(\rho=0, \mathbf{j}=0)$. Структура фотонов может быть разной. 
5. В общем случае ЭМ поле описывается четырьмя векторами D, E, B и H, между которыми возможны различные связи: линейные для волн и нелинейные для фотонов и частиц. ЭМ поле может существовать в разных состояниях, например, в состоянии ЭМ волны, фотона, нейтрино, электрона, протона, $\mu$-, $\pi$-, $K$-мезонов и т. П. Эти состояния отличаются друг от друга разными функциями зависимости плотности энергии ЭМ поля $W(\mathbf{D}, \mathbf{B})$ от векторов индукции электрического $\mathbf{D}$ и магнитного В полей. Разным уравнениям состояния ЭМ поля соответствуют разные решения одного и того же уравнения (18).

6. В классической электродинамике электрическое и магнитное поле образуют единое ЭМ поле. В электродинамике неточечных частиц $[10-12,14]$ не только поля, но и порождающие эти поля заряды тоже единые, взаимосвязанные (см. связи (4)). Было бы странно, нелогично, если было бы иначе: поля едины (взаимосвязаны), а магнитные и электрические заряды были бы независящими друг от друга.

\section{Список литературы}

[1] Дирак П.А.М. Лекции по квантовой теории поля. Мир. М. 1971. C. 7, 237.

[2] Ландау Л.Д. Собрание трудов / Под ред. Е.М. Лифшица и И.М. Халатникова. М.: Наука, 1969. С. 421-424.

[3] Тамм И.Е. Собр. Научных трудов. Т. 2. Наука. М. 1976. C. 500.

[4] Физика. Энциклопедия / Под ред. Ю.В. Прохорова. Большая Российская энциклопедия, Москва. 2003. С. 944.

[5] Фейнман Р. КЭД - странная теория света и вещества. М.: Наука, 1988. С. 144.

[6] Дирак П.А.М. Пути физики. М.: Энергоатомиздат, 1983. C. 88.

[7] Гейзенберг В.К. // УФН 1977. Т. 121. С. 657; 1977. Т. 121. C. 669.

[8] Лаптухов А.И. // Изв. Вузов. Физика. 1991. № 9. С. 26-29.

[9] Лаптухов А.И. // Изв. Вузов. Физика. 1993. № 11. C. $71-76$.

[10] Лаптухов А.И. Электродинамика и динамика неточечных частиц - новая физика микромира. М.: Тровант, 2007. C. 64.

[11] Лаптухов А.И. // Инженерная физика. 2011. № 7. C. $56-60$.

[12] Лаптухов А.И. // Инженерная физика. 2012. № 4. C. $20-30$

[13] Лаптухов А.И. // Инженерная физика. 2013. № 8. C. $16-22$.

[14] Лаптухов А.И. // Инженерная физика. 2014. № 9. С. 9-16.

[15] Ландау Л.Д, Лифшии, Е.М. Электродинамика сплошных сред. Теоретическая физика. Т. VIII. М.: Наука, 1982. C. 620 .

[16] Компанееи, А.С. Курс теоретической физики. Т. 2. Статистические законы. М.: Просвещение, 1975. С. 480.

[17] Берестеикий В.Б., Лифбии Е.М., Питаевский Л.П. Квантовая электродинамика. М.: Наука, 1980. С. 704.

[18] Денисов В.И. Лекции по электродинамике. М.: УНЦ ДО, 2007. C. 271.

http://hep.phys.msu.ru/about/personal/denisov/denisov.phtml
[19] Born M., Infield L. // Proc. Roy. Soc. 1934. Vol. A144. P. 425-430.

[20] Владимиров В.С. Уравнения математической физики. М.: Наука, 1967. C.436.

[21] Burke D.L. Field R.C., Horton-Smith G., Spencer J.E., Walz D. // Phys. Rev. Lett. 1997. Vol. 79. P. 1626-1628. 